Brazilian Journal

of Chemical

\title{
A STUDY OF THE PNEUMATIC CONVEYING OF NON-SPHERICAL PARTICLES IN A TURBULENT HORIZONTAL CHANNEL FLOW
}

\author{
S. Laín ${ }^{1 *}$ and M. Sommerfeld ${ }^{1,2}$ \\ ${ }^{1}$ Grupo de Investigación en Mecánica de Fluidos, Energetics and Mechanics Department, \\ Universidad Autónoma de Occidente (UAO), Colombia. \\ E-mail:slain@uao.edu.co \\ ${ }^{2}$ Institute für Verfahrenstechnik, Zentrum für Ingenieurwissenschaften, \\ Martin-Luther-Universität Halle-Wittenberg, \\ D06099 Halle (Saale), Germany. \\ E-mail: martin.sommerfeld@iw.unihalle.de
}

(Received: October 04, 2005 ; Accepted: March 10, 2007)

\begin{abstract}
In this work, the pneumatic conveying of non-spherical isometric particles with different degrees of non-sphericity is studied. The solids mass loading fraction is small enough in order to have a dilute flow, so inter-particle collisions can be neglected. As a first approximation, only the aerodynamic drag force acting on the particles is considered, neglecting the lift forces and the particle rotation. The drag coefficient is calculated using the correlations of Haider and Levenspiel (1989) and Ganser (1993). The numerical simulations are compared with experimental data in a narrow six meters long horizontal channel flow laden with quartz and duroplastic particles with mean diameters of 185 and $240 \mu \mathrm{m}$, respectively (Kussin, 2004).

Keywords: Turbulence; Two-phase flow; Non-spherical particles; Lagrangian approach.
\end{abstract}

\section{INTRODUCTION}

Pneumatic conveying of solid particles in channel or pipe flows is of great technical importance and is characterised by particle phase segregation due to gravity and particle inertia. Several transport regimes determined by the magnitude of conveying velocity and solids mass fraction can be identified. As the conveying of solid particles in dilute regime is one of the most important technological processes in industry, there is a large number of publications in the literature devoted to it, ranging from basic research to very applied investigations. The main reason is that the flow becomes very rich and complex, even in the dilute regime. The most important physical phenomena involved are:

- Particle transport due to turbulence.
- Large velocity gradients and relevance of slipshear force.

- Magnus effect, since the particles acquire very high angular velocities after collision with a solid wall.

- Wall collision effects, which may dominate the particle motion and affect the conveying characteristics.

- Wall roughness and particle shape, which will considerably affect the wall collision process.

- As a result of segregation effects (i.e., gravitational settling or particle inertia in bends or junctions) inter-particle collisions become already important at rather low mass loading.

- The importance of flow and turbulence modulation as a function of particle size and mass loading.

*To whom correspondence should be addressed 
The majority of experimental studies use spherical particles. However, in a number of practical situations, particle shape deviates from spherical, either being irregular or having a welldefined geometry such as granulates or fibers. Therefore, since the 1920's there has been available theoretical and experimental work on this subject in the literature, starting with the theoretical studies of Jeffery (1922) and Brenner (1963,1964 a, b, c), who analytically derived the forces for different particle shapes in the Stokes regime and different flow conditions. These results were employed by Fan and Ahmadi (1995) to calculate dispersion of very small ellipsoidal particles in homogeneous and isotropic turbulence. In this case, it was found that in the Stokes regime the influence of particle shape on turbulent dispersion was not very pronounced because the particle fluctuating velocity was almost independent of the ellipsoid axis orientation.

However, in practice the Reynolds number is usually larger than one. In this case the forces due to flow, mainly the drag force, can not be calculated analytically, so it is necessary to use experimental correlations obtained from a large number of experiments with different particle shapes: thin disks (Squires and Squires, 1937); isometric particles, which are closer to the spherical shape (Pettyjohn and Christiansen, 1948); plates and cylinders (Heiss and Coull, 1952); disks (Willmarth et al., 1964) and disks and cylinders (McKay et al., 1988). In general, the deposition of particles in pipes or containers is studied. In the case of non-isometric particles dependence on the orientation is also studied (Squires and Squires, 1937; Heiss and Coull, 1952). As a result, correlations valid for defined particle shapes and orientations are obtained. Moreover, a number of experimental studies have tried to derive, based on an equivalent particle diameter and on a sphericity variable, a general correlation for the drag coefficient of all particle shapes (Haider and Levenspiel, 1989; Thomson and Clark, 1991; Swamee and Ojha, 1991; Ganser, 1993). Chhabra et al. (1999) compared the accuracy of different correlations fit to experimental data, showing that the Ganser (1993) correlation is the best against a number of experiments for different particle shapes.

In contrast, only a few numerical and experimental studies have been performed on twophase flow with non-spherical particles. The experimental research of Morikita et al. (1998) shows that the spread of a free jet laden with quartz particles (mean diameter of $150 \mu \mathrm{m}$ ) for high Reynolds number flow is clearly bigger than that corresponding to spherical particles of approximately the same size. This phenomenon is due to the irregular motion of particles through the turbulent flow. The drag force experienced by the quartz particle is stronger than that of the glass spheres, also resulting in a higher particle mean velocity, which comes closer to the gas mean velocity. In the next step, the motion of non-spherical particles in comparison with that of the spherical ones was experimentally investigated in an annular jet and in a swirling flow using LDA. There, it was also found that the non-spherical particles follow the gas flow better than the spherical ones of the same size, and therefore their mean and fluctuating velocities are closer to the gas phase values. Therefrom it was concluded that a non-spherical particle prefers to be aligned in the flow direction, so that the drag force will be maximised and it can better follow the flow (McKay et al., 1988). A different effect was found by Losenno and Easson (2001). In this case, the sedimentation of spherical and non-spherical particles was studied using PIV. The steady terminal velocity of the non-spherical particles was clearly lower than that of the spherical, for all sizes considered. This result is in agreement with the increase in the drag force for non-spherical particles (Haider and Levenspiel, 1989).

The modelling of non-isometric particles (cylinders or granulates) is more difficult. Rosendahl (1998) calculated the orientation of a cylindrical particle along its trajectory by means of the corresponding rotation. The drag and lift force components were calculated for the corresponding orientation of the cylinder. The results show that for straw particles the spread of the jet clearly increased for the spherical particles.

Particle shape strongly influences the particlewall collision process. This has been known since the numerical simulations of Tsuji et al. (1989) for a horizontal channel flow with particles whose shape deviated only slightly from the spherical. A more pronounced deviation from the spherical shape results in a more uniform particle dispersion in the channel, decreasing the deposition due to gravity. A similar effect was produced by wall roughness in the case of spherical particles (Sommerfeld and Huber, 1999; Sommerfeld, 2003). In the work of Tsuji et al. (1989), the modelling of non-spherical particle-wall collision was based on a stochastic process to define particle orientation with respect to the wall in the collision process. However, during the movement of the particle in the flow the influence of particle shape is disregarded, which is not a bad approximation for particles whose shape deviates only slightly from the spherical shape. Finally, it is necessary to point out 
that in the literature there is nearly no information (modelling and experiments) about turbulent flows laden with solids that addresses inter-particle collisions between non-spherical particles and the effect of fluid turbulence modulation.

In this work, the pneumatic conveying of isometric particles with different degrees of nonsphericity is considered. The solids mass loading fraction is small enough to have a dilute flow, so inter-particle collisions can be neglected. The numerical simulations are carried out by means of the Euler-Lagrange approach using the Reynolds stress turbulence model for the continuous phase. As a first approximation, only the aerodynamic drag force acting on the particles is considered; therefore, the lift forces and particle rotation are not taken into account. Other forces such as the Basset history term, added mass and fluid inertia are negligible for high ratios of particle to gas densities. The drag coefficient is calculated using the correlations of Haider and Levenspiel (1989) and Ganser (1993). The particle-wall collision treatment, accounting for wall roughness, is the same as that in Sommerfeld and Huber (1999). The numerical simulations are compared with experimental data on a narrow six meters long horizontal channel flow (height $35 \mathrm{~mm}$ ) laden with quartz and duroplastic particles with mean diameters of 185 and $240 \mu \mathrm{m}$, respectively (Kussin, 2004).

\section{EXPERIMENTAL SETUP}

The entire test facility is shown in Figure 1. The main component of the test facility is a horizontal channel $6 \mathrm{~m}$ in length with a height of 35 and a width of $350 \mathrm{~mm}$, so almost two-dimensional flow conditions can be established. The upper and lower channel walls are made of interchangeable stainless steel plates in order to study the effect of wall material and wall roughness on particle behaviour.
The measurements were performed close to the end of the channel at a distance of $5.8 \mathrm{~m}$ from the entrance. In order to allow optical access for the applied phase-Doppler anemometry (PDA), the side walls were made of glass plates and a glass window of 35 by $460 \mathrm{~mm}$ was inserted at the top wall. The required air flow rate was provided by two root blowers mounted in parallel with nominal flow rates of $1002 \mathrm{~m}^{3} / \mathrm{h}$ and $507 \mathrm{~m}^{3} / \mathrm{h}$, respectively. The blowers are connected to the test section using a 130 $\mathrm{mm}$ pipe. A mixing chamber for injecting the particles and a flow conditioning section, where the cross-section changes from circular to rectangular, are mounted just before the channel. Additionally, several sieves are inserted in this section in order to ensure rather homogeneous flow conditions at the entrance of the channel. In a straight section of $2 \mathrm{~m}$ before the mixing chamber a flow meter and temperature, humidity, and pressure sensors are installed. For feeding the particle material into the mixing chamber a screw feeder is used, where the particle mass flow rate can be adjusted accordingly. In order to ensure continuous particle feeding the air is injected into the mixing chamber through a converging nozzle, whereby a lower pressure is established. The resulting jet enters the exit pipe of the mixing chamber on the opposite side. A $90^{\circ}$-bend is mounted at the end of the channel which is connected to a flow passage where the cross-section changes from rectangular to circular. A flexible pipe is used for conveying the gas-particle mixture to a cyclone separator. The separated particles are reinjected into the reservoir of the particle feeder through a bucket wheel. Finally, the air from the cyclone passes through a bag filter to remove very fine particles (i.e., the tracer particles) and is then released into the environment. The test facility described above allows conveying velocities of up to $30 \mathrm{~m} / \mathrm{s}$ to be reached and mass loadings of up to 2 ( $\mathrm{kg}$ dust) $/(\mathrm{kg}$ air) to be established.

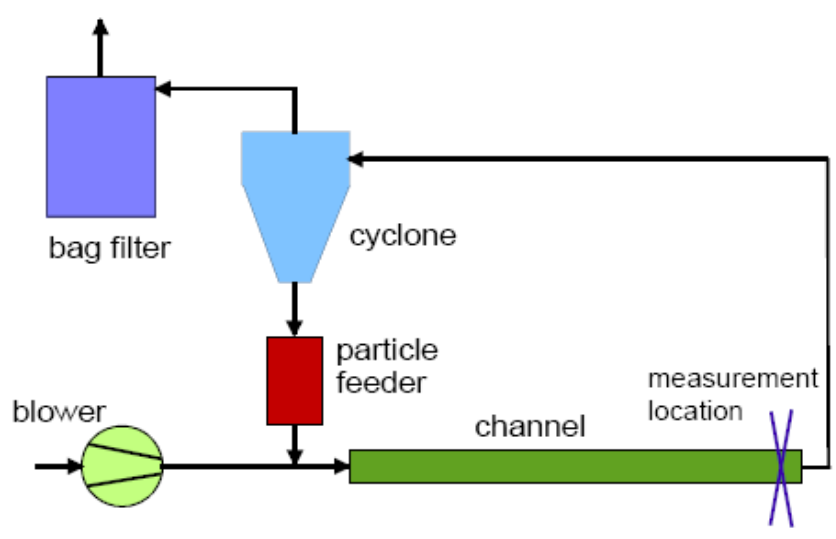

Figure 1: Schematics of the horizontal channel experimental facility. 
For the simultaneous measurement of both particle phase and continuous phase properties, PDA is applied. The PDA technique allows the timeaveraged velocities of both phases to be determined with good spatial resolution as well as the local particle size distribution. In order to allow for a measurement of the continuous phase velocities in the presence of the particles, spherical seeding particles (Ballotini 10002A) with a nominal size of about $4 \mu \mathrm{m}$ are added to the flow. This is accomplished by mixing the tracer particles with the dispersed phase particles in the reservoir of the particle feeder. The discrimination procedure is based on size measurement according to Qiu et al. (1991). The transmitting and receiving optics of the two-component PDA (Dantec Fiber PDA) is mounted on a common, computer-controlled traversing system. In order to ensure high scattering intensities for the tracer particles, the receiver is mounted at a scattering angle of $33.5^{\circ}$. At this angle, a fairly linear phase-size relation is established, as demonstrated by Sommerfeld and Tropea (1999) using Mie calculations. Vertical profiles of the properties of both phases are measured close to the end of the channel (5.8 $\mathrm{m}$ from the entrance) in the middle plane. At each measurement point, a sufficiently large number of samples (a minimum of 8000 samples for each phase) are collected to allow for statistically reliable measurements (small confidence levels of mean and rms values) of the mean and fluctuating velocity components of both phases. The complete details about the experimental rig and measurement technique can be found in Kussin (2004).

As has been said, two kinds of non-spherical isometric particles were used in the present work: quartz and duroplastic particles with mean diameters of 185 and $240 \mu \mathrm{m}$, respectively (see Figure 3). Particle sphericity $(\phi)$ is evaluated from pressure drop measurements across a fixed bed of these particles, where air flows in a laminar regime $\left(\operatorname{Re}_{\mathrm{p}}<\right.$ 10), by assuming a uniform particle arrangement in the bed. The equation of Kozeny and Carman is applied for estimating the particle sphericity (Henthorn et al., 2005).

\section{SUMMARY OF NUMERICAL APPROACH}

The numerical scheme adopted to simulate the two-phase flow developing in a horizontal channel has been described in detail previously in Laín et al. (2002). Therefore, only the basic equations and expressions will presented here in order to make clear the procedure.

The fluid flow was calculated based on the Euler approach by solving the full Reynolds stress turbulence model equations extended in order to account for the effects of the dispersed phase (Kohnen and Sommerfeld, 1997). The timedependent two-dimensional conservation equations for the fluid may be written in the general form (in tensorial notation):

$$
(\rho \phi)_{, \mathrm{t}}+\left(\rho \mathrm{U}_{\mathrm{i}} \phi\right)_{, \mathrm{i}}=\left(\Gamma_{\mathrm{ik}} \phi_{, \mathrm{k}}\right)_{, \mathrm{i}}+\mathrm{S}_{\phi}+\mathrm{S}_{\phi \mathrm{p}}
$$

where $\rho$ is the fluid density, $U_{i}$ are the Reynoldsaveraged velocity components, and $\Gamma_{\mathrm{ik}}$ is an effective transport tensor. The usual source terms within the continuous phase equations are summarised in $S_{\phi}$, while $S_{\phi p}$ represents the additional source term due to phase interaction. Table 1 summarises the meaning of this quantities for the different variables $\phi$, where $\mathrm{P}$ is the mean pressure, $\mu$ the gas viscosity and $\mathrm{R}_{\mathrm{jl}}=$ $\overline{u^{\prime}{ }_{j} u_{1}^{\prime}}$ the components of the Reynolds stress tensor.

Simulation of the particle phase by the Lagrangian approach requires solution of the equation of motion for each computational particle. This equation includes all the relevant forces to be studied, namely the drag and gravity-buoyancy forces. As stated previously, the lift forces are not taken into account at this stage and the Basset history term, the added mass and the fluid inertia are negligible for high ratios of particle to gas densities. Hence, the equations of motion for the particles are given by:

Table 1: Summary of terms in the general equation for the different variables that describe the gas phase.

\begin{tabular}{|c|c|c|}
\hline$\phi$ & $\Gamma_{i k}$ & $S_{\phi}$ \\
\hline 1 & 0 & 0 \\
\hline$\overline{\mathrm{U}_{\mathrm{j}}}$ & $\mu \delta_{\mathrm{ik}}$ & $\begin{array}{r}-\mathrm{P}_{, \mathrm{j}}+\left(\Gamma_{\mathrm{jk}} \mathrm{U}_{\mathrm{i}, \mathrm{k}}\right)_{, \mathrm{i}} \\
-\rho \mathrm{R}_{\mathrm{ij}, \mathrm{i}}+\rho \mathrm{g}_{\mathrm{j}}\end{array}$ \\
\hline $\mathrm{R}_{\mathrm{jl}}$ & $\begin{array}{cc}\mathrm{c}_{\mathrm{S}} \rho & \mathrm{R}_{\mathrm{ik}} \mathrm{k} / \varepsilon\end{array}$ & $\mathscr{P}_{\mathrm{j} 1}-\varepsilon_{\mathrm{j} 1}+\Pi_{\mathrm{j} 1}$ \\
\hline$\varepsilon$ & $\mathrm{c}_{\varepsilon} \rho \quad \mathrm{R}_{\mathrm{ik}} \mathrm{k} / \varepsilon$ & $\mathrm{c}_{\varepsilon 1} \mathscr{P}_{\mathrm{kk}} \varepsilon / \mathrm{k}-\rho \mathrm{c}_{\varepsilon 2} \varepsilon^{2} / \mathrm{k}$ \\
\hline
\end{tabular}




$$
\begin{array}{r}
\mathscr{P}_{\mathrm{jl}}=-\rho\left(\mathrm{R}_{\mathrm{jk}} \mathrm{U}_{1, \mathrm{k}}+\mathrm{R}_{\mathrm{lk}} \mathrm{U}_{\mathrm{j}, \mathrm{k}}\right) \\
\varepsilon_{\mathrm{j} 1}=\frac{2}{3} \rho \delta_{\mathrm{j} 1} \varepsilon \\
\Pi_{\mathrm{j} 1}=-\mathrm{c}_{1} \rho \frac{\varepsilon}{\mathrm{k}}\left(\mathrm{R}_{\mathrm{j} 1}-\frac{1}{3} \delta_{\mathrm{j} 1} \mathrm{R}_{\mathrm{kk}}\right)-\mathrm{c}_{2} \rho\left(\mathscr{P}_{\mathrm{j} 1}-\frac{1}{3} \delta_{\mathrm{jl}} \mathscr{\mathscr { k }}_{\mathrm{kk}}\right) \\
\mathrm{c}_{\mathrm{S}}=0.22 \quad \mathrm{c}_{\varepsilon}=0.18 \quad \mathrm{c}_{\varepsilon 1}=1.45 \\
\mathrm{c}_{\varepsilon 2}=1.9 \quad \mathrm{c}_{1}=1.8 \quad \mathrm{c}_{2}=0.6
\end{array}
$$

$$
\frac{d x_{p i}}{d t}=u_{p i}
$$

$$
\mathrm{m}_{\mathrm{p}} \frac{\mathrm{du} \mathrm{p}_{\mathrm{i}}}{\mathrm{dt}}=\frac{3}{4} \frac{\rho}{\mathrm{D}_{\mathrm{p}}} \mathrm{m}_{\mathrm{p}} \mathrm{c}_{\mathrm{D}}\left(\mathrm{u}_{\mathrm{i}}-\mathrm{u}_{\mathrm{p} \mathrm{i}}\right)
$$$$
\left|\overrightarrow{\mathrm{u}}-\overrightarrow{\mathrm{u}_{\mathrm{B}}}\right|+\mathrm{m}_{\mathrm{p}} \mathrm{g}_{\mathrm{i}}\left(1-\frac{\rho}{\rho_{\mathrm{p}}}\right)
$$

Here, $x_{p i}$ are the coordinates of the particle position,

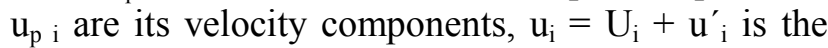
instantaneous velocity of the gas, $D_{p}$ is the particle diameter, $\rho_{p}$ is the density of the solids and $m_{p}=$ $(\pi / 6) \rho_{\mathrm{p}} \mathrm{D}_{\mathrm{p}}{ }^{3}$ is the non-spherical particle mass. The drag coefficient for the non-spherical particles is obtained using the correlations of Haider and Levenspiel (1989) and Ganser (1993). The Haider and Levenspiel correlation reads:

$$
\mathrm{c}_{\mathrm{D}}^{\mathrm{HL}}=\frac{24}{\operatorname{Re}_{\mathrm{p}}}\left(1+\mathrm{ARe_{ \textrm {p } } ^ { \mathrm { B } }}\right)+\frac{\mathrm{C}}{1+\mathrm{D} / \mathrm{Re}_{\mathrm{p}}}
$$

where $\mathrm{Re}_{\mathrm{p}}=\rho \mathrm{D}_{\mathrm{p}}\left|\overrightarrow{\mathrm{u}}-\overrightarrow{\mathrm{u}_{\mathrm{p}}}\right| / \mu$ is the particle Reynolds number and the coefficients depend on sphericity $\phi$, defined as the ratio between the surface of a volume equivalent spherical particle and the surface of the non-spherical particle. The coefficients are written as:

$$
\begin{aligned}
& A=\exp \left(2.3288-6.4581 \phi+2: 4486 \phi^{2}\right) \\
& B=0.0964+0.5565 \phi \\
& C=\exp \left(4.905-13.8944 \phi+18.4222 \phi^{2}-10.2599 \phi^{3}\right) \\
& D=\exp \left(1.4681+12.2584 \phi-20.7322 \phi^{2}+15.8855 \phi^{3}\right)
\end{aligned}
$$

The Ganser (1993) correlation distinguishes between isometric and non-isometric particles. In this work only isometric particles are used; therefore, this correlation is written as

$$
\begin{aligned}
& \mathrm{c}_{\mathrm{D}}^{\mathrm{G}}=\frac{24}{\mathrm{~K}_{1} \mathrm{~K}_{2} \mathrm{Re}_{\mathrm{p}}}\left(1+0.118\left(\mathrm{~K}_{1} \mathrm{~K}_{2} \mathrm{Re}_{\mathrm{p}}\right)^{0.6567}\right)+ \\
& \frac{0.4305}{1+\frac{3305}{\mathrm{~K}_{1} \mathrm{~K}_{2} \mathrm{Re}_{\mathrm{p}}}}
\end{aligned}
$$

where the Stokes, $\mathrm{K}_{1}$, and Newton, $\mathrm{K}_{2}$, shape factors are given for isometric particles as

$$
\begin{aligned}
& \mathrm{K}_{1}=\left(\frac{1}{3}+\frac{2}{3 \sqrt{\phi}}\right) \\
& \mathrm{K}_{2}=10^{1.8148(-\log \phi)^{0.5743}}
\end{aligned}
$$

Both drag coefficient correlations are shown in Figure 2. It can be seen that the drag coefficient increases continuously as the sphericity $\phi$ decreases and that even for $\phi=0.9$ the deviation from the spherical drag law is noticeable for Reynolds numbers larger than 100 .

The equations to calculate particle motion are solved by numerical integration of the differential equations (Eqs. 2-3). For sufficiently small time steps and assuming that the forces remain constant during this time step, the new particle location and velocities are calculated. The time step for the particle tracking is chosen to be $50 \%$ of the smallest of all relevant time scales, such as the particle relaxation time, the integral time scale of turbulence and the time required by the particle to cross a control volume. 
When a particle collides with a wall, the wall collision model provides the new particle velocities and the new location in the computational domain after rebound. As stated in the introduction, in a horizontal channel flow the sedimentation of non-spherical particles is stronger than that of spherical ones. Therefore, the isometric non-spherical particle - wall collision process will be simulated as a spherical particlerough wall collision, whose roughness varies according to the degree of particle non-sphericity. The wall collision model applied is described in
Sommerfeld and Huber (1999); the wall roughness experienced by the particle is simulated assuming that the impact angle is composed of the particle trajectory angle plus a stochastic contribution due to wall roughness, $\Delta \gamma$, which depends on the structure of wall roughness and particle size. Moreover, according to the experimental results of Sommerfeld and Huber (1999), the restitution $\left(\mathrm{e}_{\mathrm{w}}\right.$, defined as the absolute value of the normal velocity components after collision to that before collision) and friction $\left(\mu_{\mathrm{w}}\right)$ coefficients vary with the impact angle $\alpha$.

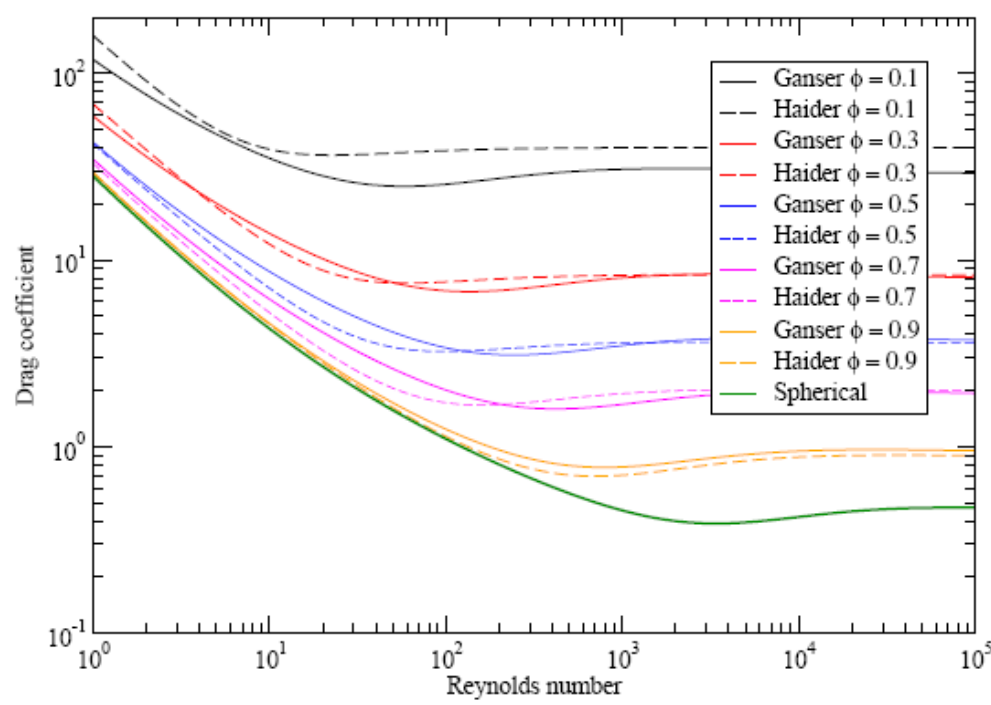

Figure 2: Drag laws of Ganser and Haider and Levenspiel depending on the sphericity $\phi$ (isometric particles)

\section{INFLUENCE OF PARTICLES ON THE CARRIER FLOW}

The standard expression for the momentum equation source term due to the particles has been used. It is obtained by time and ensemble averaging for each control volume in the following form:

$$
\begin{aligned}
& \overline{\mathrm{S}_{\mathrm{U}_{\mathrm{i}} \mathrm{p}}}=-\frac{1}{\mathrm{~V}_{\mathrm{cv}}} \sum_{\mathrm{k}} \mathrm{m}_{\mathrm{k}} \mathrm{N}_{\mathrm{k}} \times \\
& \sum_{\mathrm{n}}\left\{\left(\left[\mathrm{u}_{\mathrm{pi}}\right]_{\mathrm{k}}^{\mathrm{n}+1}-\left[\mathrm{u}_{\mathrm{pi}}\right]_{\mathrm{k}}^{\mathrm{n}}\right)-\mathrm{g}_{\mathrm{i}}\left(1-\frac{\rho}{\rho_{\mathrm{P}}}\right) \Delta \mathrm{t}_{\mathrm{L}}\right\}
\end{aligned}
$$

where the sum over $\mathrm{n}$ indicates averaging along the particle trajectory (time averaging) and the sum over $\mathrm{k}$ is related to the number of computational particles passing the considered control volume with the volume $\mathrm{V}_{\mathrm{cv}}$. The mass of an individual particle is $\mathrm{m}_{\mathrm{k}}$ and $\mathrm{N}_{\mathrm{k}}$ is the number of real particles in one computational particle. $\Delta \mathrm{t}_{\mathrm{L}}$ is the Lagrangian time step which is used in the solution of (3).

The source terms in the conservation equations of the Reynolds stress components, $\mathrm{R}_{\mathrm{jl}}$, are expressed in the Reynolds average procedure as:

$$
S_{R_{j l} p}=\overline{u_{j} S_{U_{l} p}}+\overline{u_{1} S_{U_{j} p}}-\left(U_{j} \overline{S_{U_{l} p}}+U_{1} \overline{S_{U_{j} p}}\right)
$$

while the source term in the $\varepsilon$-equation is modelled in the standard way:

$$
\mathrm{S}_{\varepsilon \mathrm{p}}=\mathrm{C}_{\varepsilon 3} \frac{1}{2} \frac{\varepsilon}{\mathrm{k}} \mathrm{S}_{\mathrm{R}_{\mathrm{jj}} \mathrm{p}}
$$

with $\mathrm{C}_{\varepsilon 3}=1.0$ and the sum is implicit in the repeated subindex $\mathrm{j}$. 
All the calculations were performed with a mesh of $960 \times 40$ control volumes in the horizontal $(6000$ $\mathrm{mm})$ and vertical $(35 \mathrm{~mm})$ directions, respectively. This resolution is found to be sufficient for producing grid-independent results. A converged solution of the coupled two-phase flow system is obtained by successive solution of the Eulerian and Lagrangian part, respectively. Initially, the flow field is calculated without particle phase source terms until a converged solution is achieved. Thereafter, a large number of parcels are tracked through the flow field (typically 20,000) and the source terms are sampled. Moreover, for each control volume the particle concentration, the local particle size distribution and the size-velocity correlations for the mean velocities and the rms values are sampled. From the second Eulerian calculation, the source terms of the dispersed phase are introduced using an under-relaxation procedure (Kohnnen et al., 1994).

For the present calculations, characterised by a small particle mass loading ratio, typically about 10 coupling iterations with an under-relaxation factor of 0.5 are necessary in order to achieve convergence.

\section{RESULTS}

All the results presented were obtained for an average air velocity of about $20 \mathrm{~m} / \mathrm{s}$. Two kinds of isometric non-spherical particles were considered in this study (Figure 3):

1. Quartz particles, with mean diameter $D_{p}=185$ $\mu \mathrm{m}$, density $\rho_{\mathrm{p}}=2650 \mathrm{~kg} / \mathrm{m}^{3}$ and measured sphericity $\phi$ of around 0.7 .

2. Duroplastic particles, with mean diameter $D_{p}=$ $240 \mu \mathrm{m}$, density $\rho_{\mathrm{p}}=1480 \mathrm{~kg} / \mathrm{m}^{3}$ and measured sphericity $\phi$ of around 0.6 .
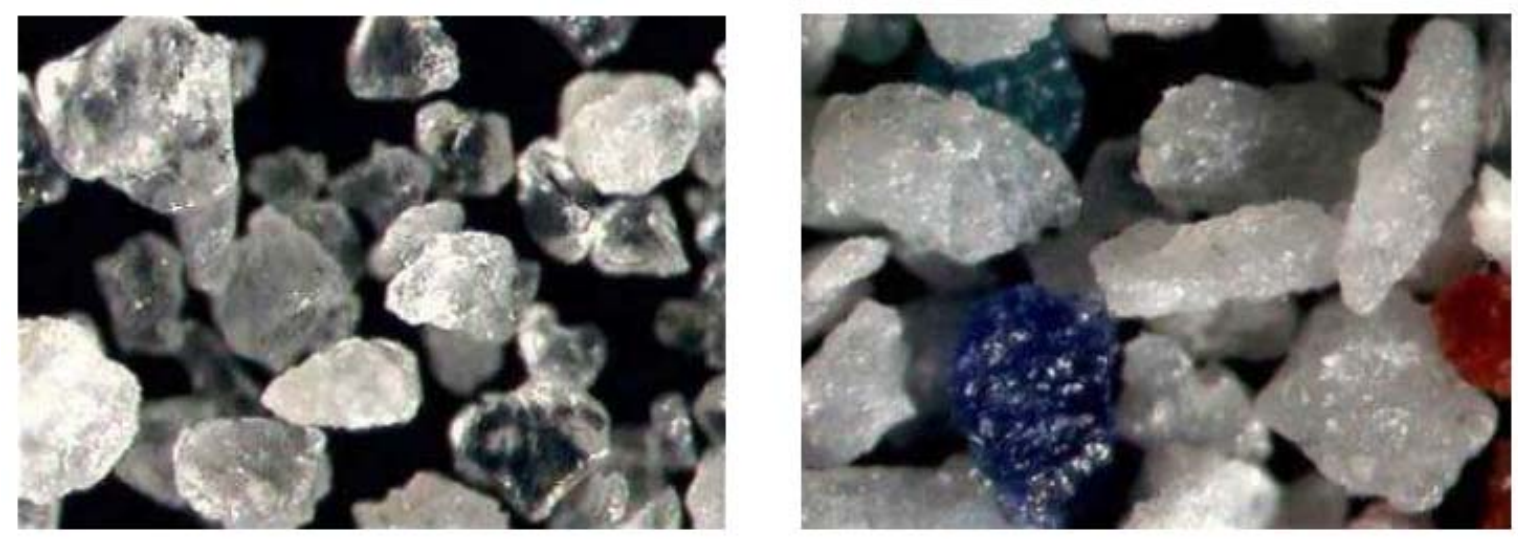

Figure 3: Quartz particles, $D_{p}=185 \mu \mathrm{m}$ (left) and duroplastic particles, $D_{p}=240 \mu \mathrm{m}$ (right).

\section{Results for the Quartz Particles}

In this section, the results for quartz, isometric, non-spherical particles $\left(D_{p}=185 \mu \mathrm{m}\right)$ are presented. The results were obtained for a particle mass fraction $\eta=0.1$, the turbulence modulation was taken into account by means of two-way coupling and nonspherical particle-wall interaction was treated in the same way as it was for spherical particles using a stochastic contribution to the impact angle $\Delta \gamma$ (Sommerfeld and Huber, 1999). Finally, no lift forces (slip shear and slip rotational lift) were considered.

Figure 4 illustrates the effect of the drag law on the mean and fluctuating velocities of the particle phase for two values of sphericity $\phi=0.6,0.8$, and $\Delta \gamma=1.5^{\circ}$. Due to the low particle mass fraction, the gas turbulence modulation is fairly small and the results for the gas phase are very similar in all cases; therefore, only one line will be plotted showing agreement with the measurements. In the particles, however, the effect of the different drag laws can be seen, as expected. Accordingly, the lower the value of $\phi$ the higher is the drag coefficient, and the particle mean velocity (Figure 4, top left) comes closer to that of the gas phase. The effect of sphericity on the rms values of particle axial velocity is not too large, but for the particle vertical rms values it can be clearly seen that the smaller the $\phi$, the lower the value of $v_{p}$. This is true for both drag formulations, Haider and Levenspiel and Ganser. Also the v', values are slightly lower for the Ganser correlation than those for the Haider and Levenspiel correlation, also resulting in higher values for the axial mean velocity in the case of the Ganser correlation. However, the experimental v' ${ }_{p}$ values are lower than the values in the numerical results. Regarding the particle number flux, the drag law and sphericity have nearly no effect, as can be seen below (Figure 5, bottom right). Moreover, the calculations compare fairly well with the experimental particle concentration profile. 

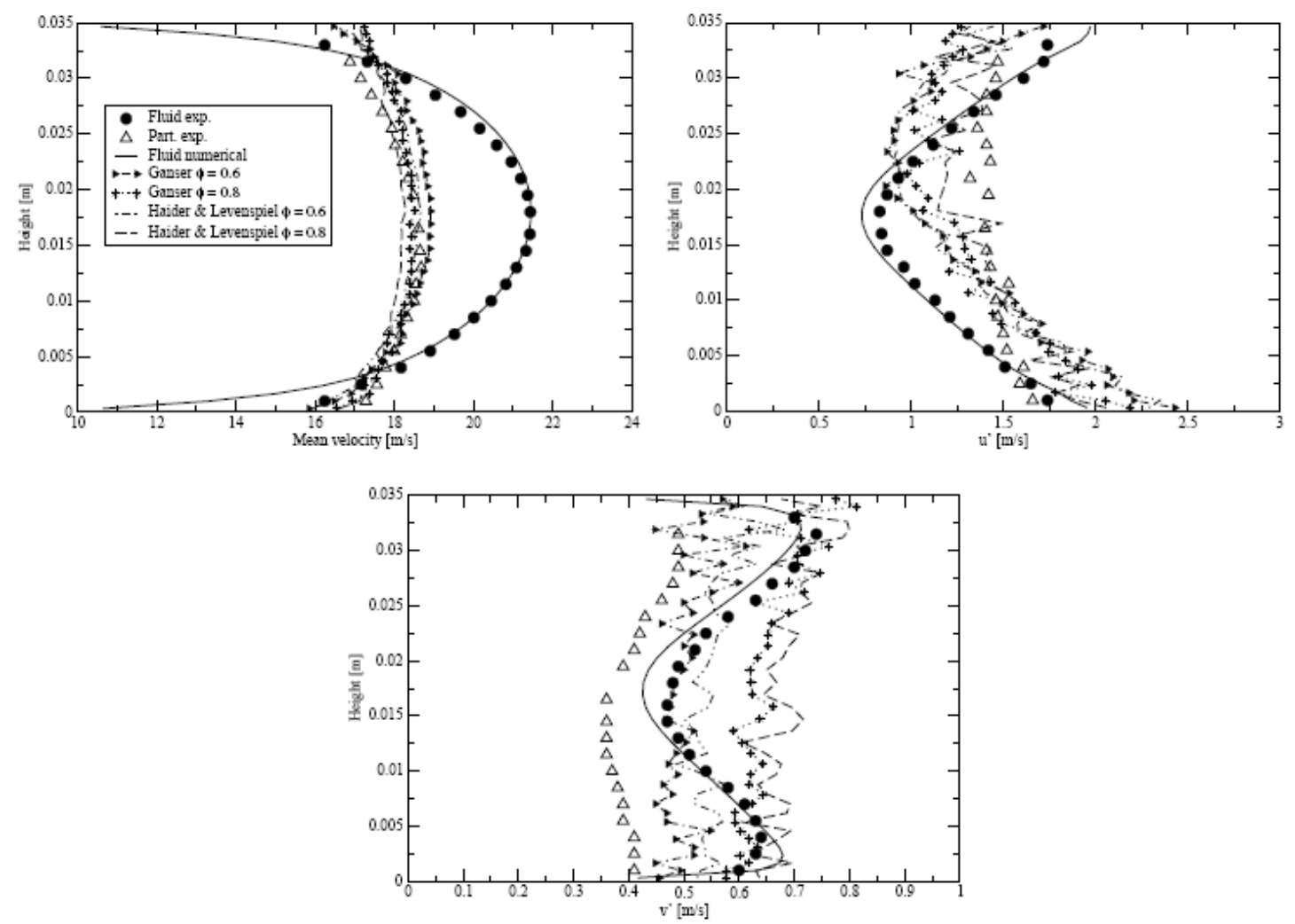

Figure 4: Mean and fluctuating velocities for the drag laws of Ganser and Haider and Levenspiel, depending on the sphericity $\phi$ (isometric quartz particles): mean horizontal velocity (top left), horizontal rms velocity (top right) and vertical rms velocity (bottom).

Additionally in Figure 5 the effect of wall roughness is shown for the cases of $\Delta \gamma=1.5^{\circ}$ and $\Delta \gamma=3^{\circ}$ for the fixed particle sphericity $\phi=0.8$ and with both drag correlations. The effects are very similar to what happens with spherical particles. The particle mean velocity decreases for the highest roughness due to the increase in wall collision frequency (Figure 5, top left), which results in more uniform particle concentration profiles (Figure 5, bottom right), and the particle fluctuating velocity increases noticeably in both components (horizontal and vertical) due to the resuspension effect of wall roughness. The particle concentration profiles for the higher wall roughness value of $\Delta \gamma=3^{\circ}$ are consistently more uniform than that for $\Delta \gamma=1.5^{\circ}$, also due to the improvement in particle resuspension by increasing wall roughness. However, the use of $\Delta \gamma=3^{\circ}$ is discouraged due to the high values provided for the particle fluctuating velocities and also because the choice of $\Delta \gamma=1.5^{\circ}$ fit well the experimental points.

In Figures 4 and 5 it can be seen that the effect of wall roughness on particle velocity is more pronounced than the effect of sphericity. This fact is not surprising due to the enormous importance of wall roughness in particle redispersion, even with spherical particles, as discussed in much detail in Sommerfeld (2003).

On the other hand, the effect of friction and wall restitution is shown in Figure 6. The effect of wall restitution $e_{w}$ is quite small and the changes are mainly due to wall friction $\mu_{\mathrm{w}}$. Two cases were considered with sphericity $\phi=0.8$ and $\Delta \gamma=1.5^{\circ}$ :

1. $\mu_{\mathrm{w}}=\max (0.5-0.0175 \alpha, 0.15)$

2. $\mu_{\mathrm{w}}=\max (0.4-0.015 \alpha, 0.1)$

where $\alpha$ is the mean impact angle of the particle trajectory intersecting the wall.

The first case has the same values as those for spherical particles, while the second case has data suggested by the experiments. As can be seen in the graphs, increasing the friction reduces the mean velocity (due to the obvious increase in friction force with the wall) and increases the particle horizontal rms value $u^{\prime}$. However, there is nearly no effect on the $\mathrm{v}_{\mathrm{p}}$ component and on the particle flux. 

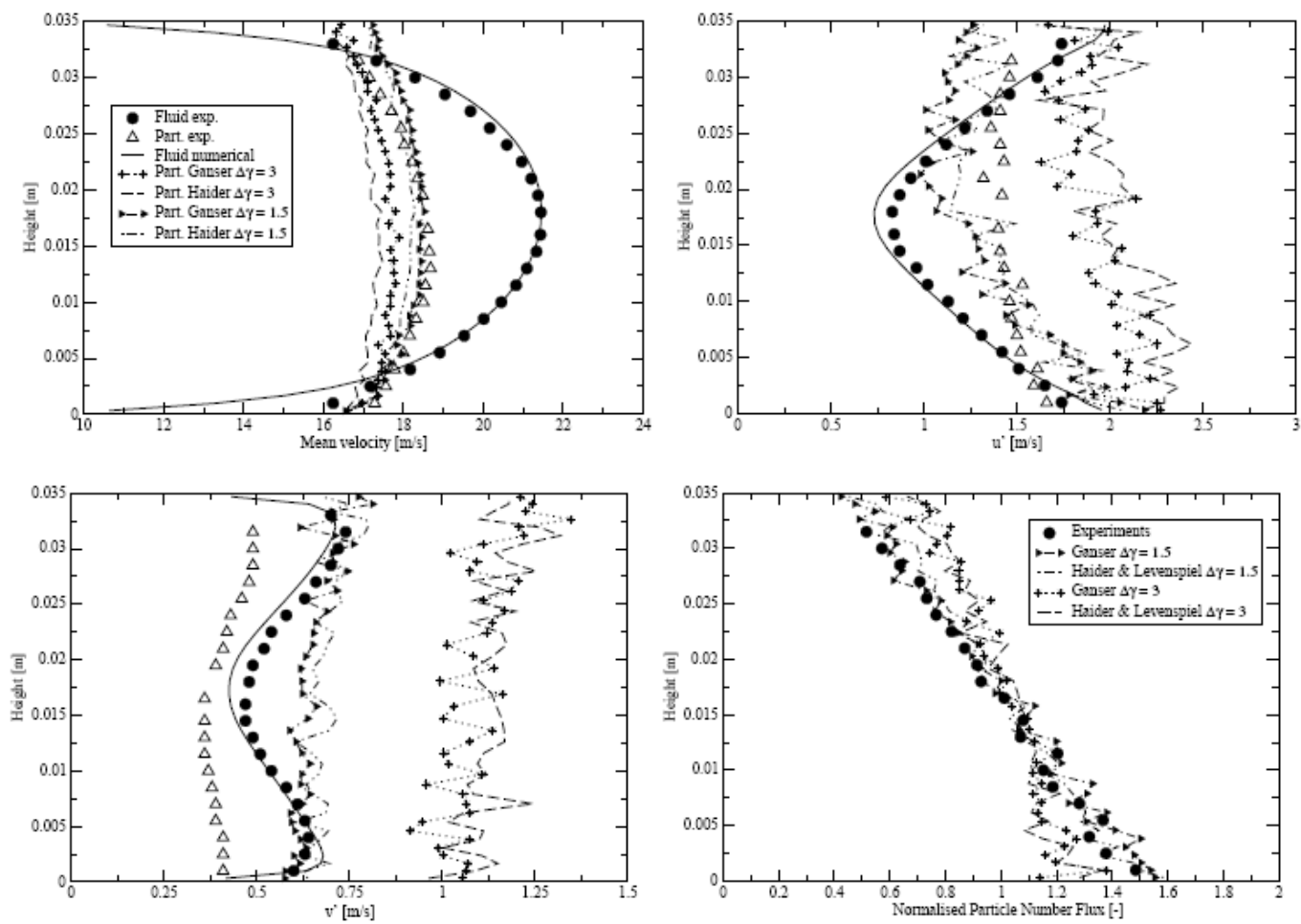

Figure 5: Mean and rms values of quartz particle velocities and normalised particle number flux for different wall roughness and the drag laws of Ganser and Haider and Levenspiel, for a sphericity of $\phi=0.8$ (isometric particles): mean horizontal velocity (top left), horizontal rms velocity (top right), vertical rms velocity (bottom left) and normalised particle number flux (bottom right).
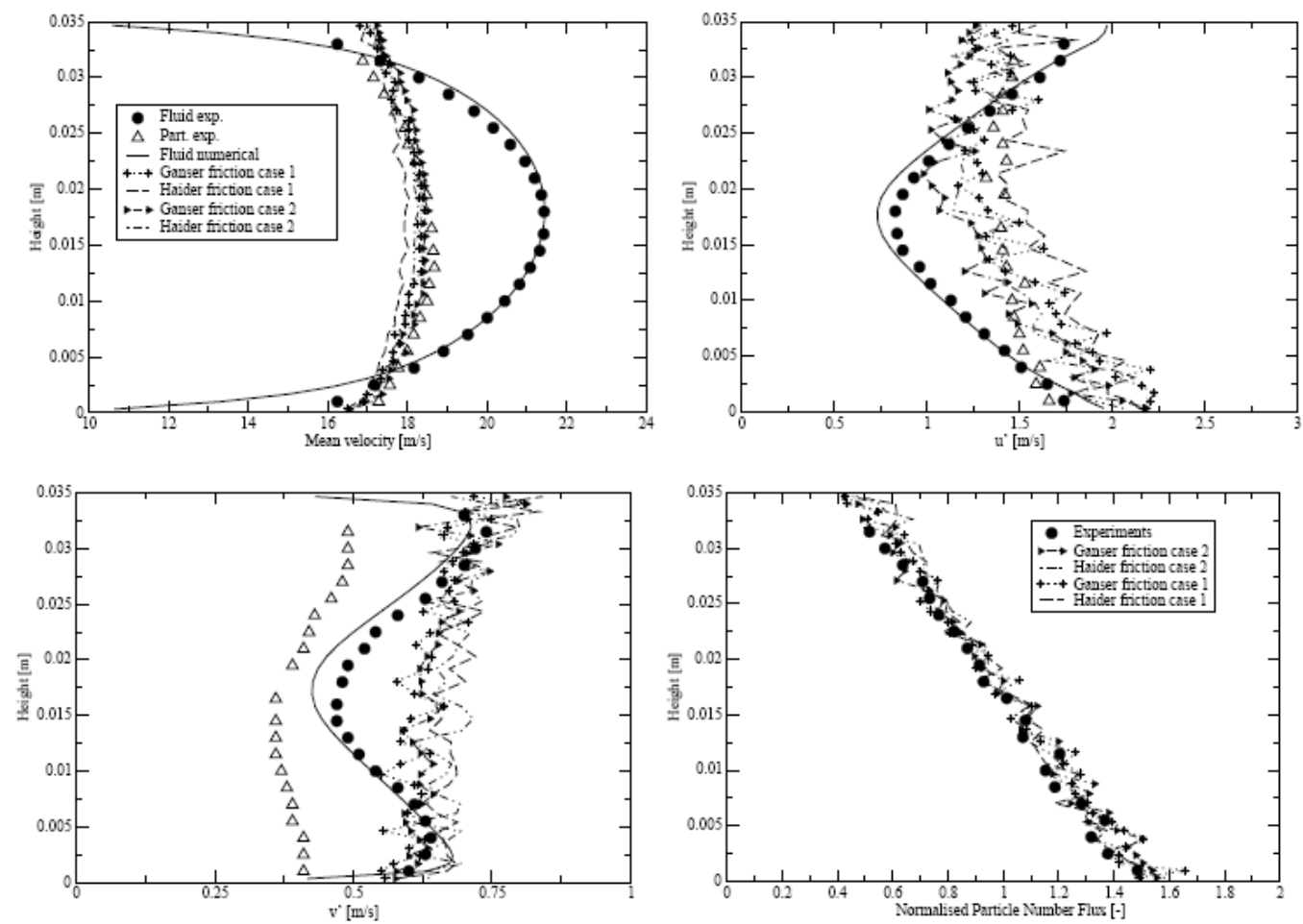

Figure 6: Mean and rms values of quartz particle velocities and normalised particle number flux for different friction cofficients and the drag laws of Ganser and Haider and Levenspiel, for a sphericity of $\phi=0.8$ (isometric particles) and $\Delta \gamma=1.5^{\circ}$ : mean horizontal velocity (top left), horizontal rms velocity (top right), vertical rms velocity (bottom left) and normalised particle number flux (bottom right). 


\section{Results for the Duroplastic Particles}

Here, the results for duroplastic, isometric, nonspherical particles $(\mathrm{Dp}=240 \mu \mathrm{m})$ with a sphericity $\phi$ of around 0.6 are presented. These results were obtained under the following conditions: mass fraction $\eta=0.06$, so the two-way coupling was nearly non-existent; as before, particle-wall interaction was treated in the same way as it is for spherical particles with a $\Delta \gamma=1.5^{\circ}$; the Ganser drag law was used with two sphericities; the friction coefficient employed was $\mu_{\mathrm{w}}=\max (0.4-0.015 \alpha$, 0.1 ) and the restitution coefficient for large impact angles was 0.52 . In these simulations the effect of sphericity as well as transverse lift forces (slip-shear and slip-rotational) is analysed. The expressions for the lift forces considered are the same as those for spherical particles (Laín et al., 2002). This choice was motivated by the lack of available correlations of the lift forces for non-spherical particles in the literature.

As in the case of quartz particles, the gas flow was nearly the same in all cases; therefore, only one line is plotted to show the agreement with the measurements (Figure 7). As observed for the quartz particles, the lower the value of $\phi$, the higher is the drag coefficient and the closer the particle mean velocity comes to that of the gas phase (Figure 7, top left). When the lift forces are considered, the particle mean velocity in the lower half of the channel is slightly increased, while both rms values are only slightly affected. The horizontal component of the particle rms value is predicted to be steeper than that observed in the experiment, and hence considerably underpredicted in the upper part of the channel (Figure 7, top right). The vertical particle rms values are underpredicted, especially for the lower sphericity value (Figure 7, bottom left). It should however be emphasised that the calculated profiles of $\mathrm{v}$ ' for the particles are lower than the values for the fluid, similar to what is seen in the experimental data. For the particle number flux, it can be seen that the lift forces drastically change the concentration profile, making it very different from that seen in the experiments (Figure 7, bottom right). Therefore, the use of the lift forces (at least the spherical version of them) should not be considered for this case of duroplastic particles.
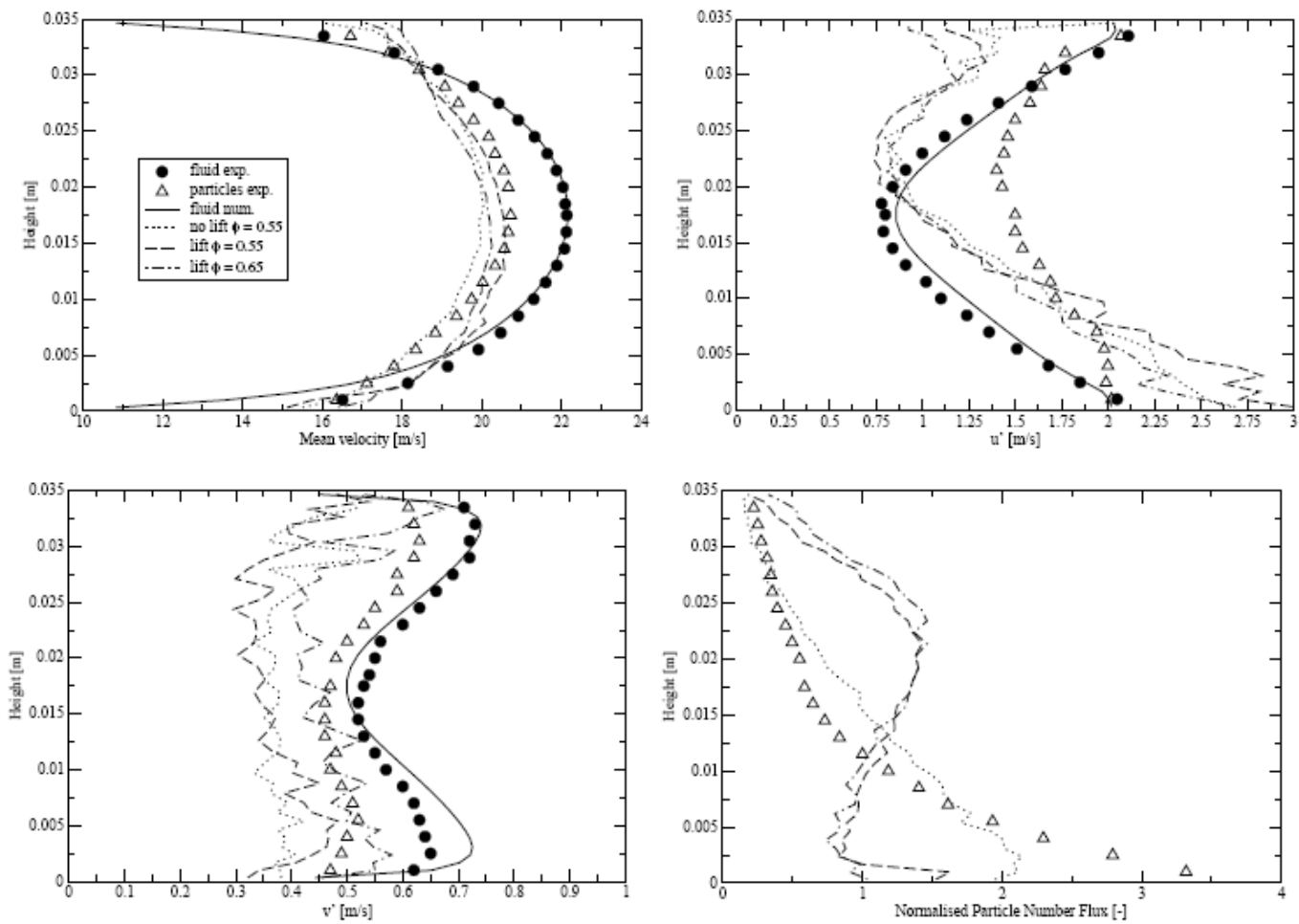

Figure 7: Mean and fluctuating velocities as well as particle number flux for duroplastic particles, influence of transverse lift forces and particle sphericity $\phi$ (isometric particles): mean horizontal velocity (top left), horizontal rms velocity (top right), vertical rms velocity (bottom left) and normalised particle number flux (bottom right). 


\section{SUMMARY AND CONCLUSIONS}

In this work, the pneumatic conveying of isometric particles with different degrees of nonsphericity, was studied. The solids mass loading fraction was small enough in order to have a dilute flow, so inter-particle collisions were neglected. The numerical simulations were carried out by means of the Euler-Lagrange approach using the Reynolds stress turbulence model for the continuous phase. As a first approximation, only the aerodynamic drag force acting on the particles was considered. The drag coefficient was calculated using the correlations of Haider and Levenspiel (1989) and Ganser (1993). Non-spherical particle-wall collision was treated similarly to spherical particle-rough wall collision described in Sommerfeld and Huber (1999). The numerical simulations were compared with experimental data on a six meters long horizontal channel flow laden with quartz and duroplastic particles with mean diameters of 185 and $240 \mu \mathrm{m}$, respectively (Kussin, 2004). As a result, the Ganser correlation provided higher particle mean velocities than the Haider and Levenspiel correlation. The results compare qualitatively well with the experiments. Good agreement is found for the horizontal particle mean velocity when the correct value for sphericity is selected. The computed profiles for both components of the particle rms values show larger deviations from the measurements. For the quartz particles the vertical rms value was considerably overpredicted for the drag law, wall roughness and sphericity considered. In the case of the duroplastic particles the predicted vertical rms values show the same trend as the experimental data. However, the slope of the horizontal rms values is not obtained correctly. In addition it was found that taken into account the transverse lift forces for spherical particles did not provide good results, especially for the profile of the particle number flux.

\section{NOMENCLATURE}

$\begin{array}{ll}c_{D} & \text { Drag coefficient } \\ D_{p} & \text { Particle diameter } \\ \mathrm{e}_{\mathrm{w}} & \text { Wall restitution coefficient } \\ & \\ \mathrm{g} & \text { Gravity acceleration } \\ \mathrm{k} & \text { Turbulent kinetic energy } \\ \mathrm{m}_{\mathrm{p}} & \text { Particle mass } \\ \mathrm{P} & \text { Pressure }\end{array}$

$\begin{array}{ll}\mathrm{R}_{\mathrm{ij}} & \text { Reynolds stress components } \\ \mathrm{Re}_{\mathrm{p}} & \text { Particle Reynolds number } \\ \mathrm{S}_{\phi} & \text { Generic source term } \\ \Delta \mathrm{t}_{\mathrm{L}} & \text { Lagrangian time step } \\ \mathrm{u} & \text { Instantaneous fluid velocity } \\ \mathrm{U} & \text { Mean fluid velocity } \\ \mathrm{u} & \text { Fluctuating velocity } \\ \mathrm{u}_{\mathrm{p}} & \text { Particle velocity } \\ \mathrm{x}_{\mathrm{p}} & \text { Particle position }\end{array}$

\section{Greek Letters}

$\alpha \quad$ Mean impact angle particle

$$
\text { trajectory }
$$

$\varepsilon \quad$ Rate of dissipation of turbulent kinetic energy

$\phi \quad$ Particle sphericity

$\Delta \gamma \quad$ Variance of the impact angle due to wall roughness

$\eta \quad$ Loading ratio

$\mu \quad$ Fluid viscosity

$\mu_{\mathrm{w}} \quad$ Wall friction coefficient

$\rho \quad$ Fluid density

$\rho_{\mathrm{p}} \quad$ Particle density

\section{ACKNOWLEDGEMENT}

The financial support of the Vicerrectoría de Investigaciones y Desarrollo Tecnológico of the Universidad Autónoma de Occidente through the project "Estudio Experimental y Modelización del Movimiento de Partículas no Esféricas en un Flujo Turbulento y su Interacción con Paredes Sólidas" is gratefully acknowledged.

\section{REFERENCES}

Brenner, H., The Stokes resistance of an arbitrary particle (I). Chemical Engineering Science, Vol. 18, 1-25 (1963).

Brenner, H., The Stokes resistance of an arbitrary particle (II) An extension. Chemical Engineering Science, Vol. 19, 599-629 (1964 a).

Brenner, H., The Stokes resistance of an arbitrary particle (III) Shear fields. Chemical Engineering Science, Vol. 19, 631-651 (1964 b).

Brenner, H., The Stokes resistance of an arbitrary particle (IV) Arbitrary fields of flow. Chemical Engineering Science, Vol. 19, 703-727 (1964 c).

Chhabra, R.P., Agarwal, L. and Sinha, N.K., Drag on non-spherical particles: an evaluation of available 
methods. Powder Technology, Vol. 101, 288-295 (1999).

Fan, F.G. and Ahmadi, G., Dispersion of ellipsoidal particles in an isotropic pseudoturbulent flow field. Trans. ASME, J. Fluids Eng., Vol. 117, 154-161 (1995).

Ganser, G. H., A rational approach to drag prediction of spherical and nonspherical particles. Powder Technology, Vol. 77, 143-152 (1993).

Haider, A. and Levenspiel, O., Drag coefficient and terminal velocity of spherical and nonspherical particles. Powder Technology, Vol. 58, 63-70 (1989).

Heiss, J. F. and Coull, J., The effect of orientation and shape on the settling velocity of nonisometric particles in a viscous medium. Chemical Engineering Progress, Vol. 48, 133-140 (1952).

Henthorn, K. H., Park, K. and Curtis J. S., Measurement and prediction of pressure drop in pneumatic conveying: Effect of particle characteristics, mass loading, and Reynolds number. Industrial and Engineering Chemistry Research, Vol. 44, 5090-5098 (2005).

Jeffery, G., The motion of ellipsoidal particles immersed in a viscous fluid. Proceedings of the Royal Society, Vol. 102A, 161-179 (1922).

Kohnen, G., Rüger, M. and Sommerfeld, M., Convergence behaviour for numerical calculations by the Euler/Lagrange method for strongly coupled phases. Num. Meth. for Multiphase Flows, FED Vol. 185. Eds: Crowe et al., 191-202 (1994).

Kohnen, G. and Sommerfeld, M., The effect of turbulence modelling on turbulence modification in two-phase flows using the Euler-Lagrange approach. Proc. 11th Symp. on Turbulent Shear Flows, Grenoble (France), Vol. 2, P3, 23-28 (1997).

Kussin, J., Experimentelle Studien zur Partikelbewegung und Turbulenzmodifikation in einem horizontalen Kanal bei unterschiedlichen Wandrauhigkeiten. $\mathrm{PhD}$ Thesis (in German) Martin Luther University Halle-Wittenberg (2004).

Laín, S., Sommerfeld, M. and Kussin, J., Experimental studies and modelling of four-way coupling in particle-laden horizontal channel flow. Int. J. Heat and Fluid Flow, Vol. 23, pp. 647-656 (2002).

Losenno, C. and Easson, W. J., Free- falling of irregular particles. 4th Int. Conference of Multiphase Flow, New Orleans, ICMF 2001.
McKay, G., Murphy, W.R. and Hills, M., Settling characteristics of discs and cylinders. Chem. Eng. Res. Des., Vol. 66, 107-112 (1988).

Morikita, H., Suzuki, K., Hishida, K. and Maeda, M., Dispersion of spherical and nonspherical particles in a round jet measured by shadow Doppler velocimetry. 3rd Int. Conf. On Multiphase Flow, ICMF'98, Lyon, France (1998).

Pettyjohn, E. S. and Christiansen, E. B., Effect of particle shape on free-settling rates of isometric particles. Chemical Engineering Progress, Vol. 44, No. 2, 157-172 (1948).

Qiu, H. H., Sommerfeld, M. and Durst, F., High resolution data processing for phase - Doppler measurements in a complex two-phase flow. Meas. Sci. Tech., Vol. 2, 455-463 (1991).

Rosendahl, L.A., Extending the modelling framework for gas-particle systems: Application of multiparameter shape descriptions to nonconventional solid fuels in reacting and nonreacting environment. $\mathrm{Ph}$. D. Thesis, Institute of Energy Technology, Aalborg University, Denmark (1998).

Sommerfeld, M., and Huber, N., Experimental analysis and modelling of particle-wall collision. Int. J. Multiphase Flow, Vol. 25, pp. 1457-1489 (1999).

Sommerfeld, M. and Tropea, C., Single-point laser measurement. In: Soo S. L. (ed.) Instrumentation for fluid-particle flow, Chap. 7, Noyes Publications, Park Ridge N.J., pp 252-317 (1999).

Sommerfeld, M., Analysis of collision effects for turbulent gas-particle flow in a horizontal channel: Part I Particle transport. Int. J. Multiphase Flow, Vol. 29, 675-699 (2003).

Squires, L. and Squires, Jr. W., The sedimentation of thin discs. Trans. Am. Inst. Chem. Eng., Vol. 33, 1-12 (1937).

Swamee, P.K. and Ojha, C.A.P., Drag coefficient and fall velocity of nonspherical particles. J. of Hydraulic Engineering, Vol. 117, 660-667 (1991).

Thompson, T. L. and Clark, N.N., A holistic approach to particle drag prediction. Powder Technology, Vol. 67, 57-66 (1991).

Tsuji, Y., Shen, N.Y., and Morikawa, Y., Numerical simulation of gas-solid flows I: Particle-to-wall collisions. Tech. Reports of the Osaka University, Vol. 39, pp. 233- 241 (1989).

Willmarth, W. W., Hawk, N. E. and Harvey, R. L., Steady and unsteady motions and wakes of freely falling disks. Physics of Fluids, Vol. 7, 197-208 (1964). 